\title{
Highly Collimated Backlight for Liquid Crystal Displays
}

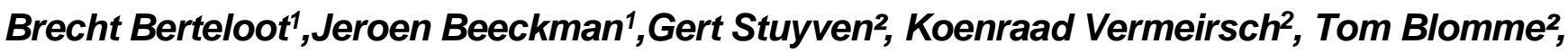 \\ Kristiaan Neyts ${ }^{1}$ \\ 'Liquid Crystals and Photonics Group, Ghent University, Belgium \\ 2ScioTeq, Belgium \\ Contact Author Email: brecht.berteloot@ugent.be
}

\begin{abstract}
In this work, we present the design of a collimated backlight design that can be used for a liquid crystal display. The design is based on a hexagonal array of white light emitting diodes (LEDs) in combination with two arrays of lenses. Ray tracing simulations show a full width - half maximum (FWHM) collimation angle of $2.8^{\circ}$ with a uniformity of $73 \%$. A prototype of this design has been fabricated and its performance has been analyzed. Compared to a standard backlight, the collimated backlight provides a 4.2 times better contrast ratio for a twisted nematic (TN) liquid crystal (LC) panel. In combination with a diffusor in front of the $L C$ panel, the contrast ratio under a wide viewing angle is improved by a factor of 10 and the color shift is reduced by $79 \%$.
\end{abstract}

\section{Author Keywords}

Collimation; Collimated backlight; Directional backlight; Liquid Crystal Display; Color shift; Contrast ratio; Twisted Nematic LCD

\section{Introduction}

The two main components of a liquid crystal display (LCD) are the white backlight and the liquid crystal panel that spatially modulates the intensity of the white light and filters out red green or blue light, based on an electronic image.

The two most common types of backlights are direct-lit and edge-lit backlights. In a direct-lit backlight, an two-dimensional array of LEDs are placed directly behind the area of the LC panel. In an edge-lit backlight, a linear array of LEDs are placed in optical contact with one or more edges of a light guide plate (LGP). The light of the LEDs is waveguided within the volume of the LGP according to the principle of total internal reflection. Small, well-distributed extraction features on the LGP break the condition for total internal reflection and provide a uniform emission of the LED light. Both types of backlights are typically used in combination with a set of diffusor plates in order to emit light distributed over a wide angle, corresponding to the desired viewing angle of the liquid crystal display (LCD).

On the other hand a directional, collimated backlight can provide several advantages compared to a normal backlight with regards to contrast ratio, transmission, color, 3D-applications and energy consumption. [1,2]

In literature, directional backlights can be found which achieve a degree of collimation in the range of $15^{\circ}$ down to $4.9^{\circ}$ (FWHM). This high degree of collimation is usually only obtained along one axis of the display, while for the other axis, the collimation is at least $20^{\circ}$ (FWHM). [1,2,3] A high degree of collimation can be achieved by making use of compound parabolic concentrators (CPCs). The degree of collimation with this approach is strongly dependent on the length of the collimator and a high degree of collimation typically requires a length of several $\mathrm{cm}$. In addition to this disadvantage, CPCs have a circular cross section which makes it impossible to place them next to each other, in order to obtain uniform collimated illumination. Due to these reasons the CPCs cannot be used for designing a collimated backlight for an LCD.[4]

In this work we propose a collimated backlight based on an hexagonal array of white LEDs. In front of each LED a combination of a small aspheric lens and a hexagonal freeform lens is used to obtain a highly collimated emission with good homogeneity.

\section{Backlight design}

The backlight is based on a hexagonal array of LEDs, as in a direct-lit backlight. The light of each LEDs is collimated by using two consecutive plano-convex lenses. The first lens has circular symmetry and is placed close to the LED to collect as much of the light of the LED as possible and collimate it in a first step. To achieve this goal we use a plano-convex lens with a low f-number with the flat side mounted closest to the LED.

The second lens is used to further collimate the light transmitted by the first small lens. As the LEDs are arranged in a hexagonal array, the maximum dimension of the second lens is limited by the hexagonal unit cell of the LED array. In order to have a homogeneous field of transmitted light, without dark regions, the lenses of the second array are cut into hexagons and tiled next to each other into a hexagonal lens array.

As the degree of collimation is limited by the conservation of étendue, it is desired to use an LED with a small emitting area, and a hexagonal unit cell with a large area. The ratio of the area of the hexagonal unit cell over the area of the LED, is limited because this ratio determines by how much the backlight is less bright than the LED.

Based on the above requirements, we selected a white LED with an emitting area of roughly $500 \mu \mathrm{m}$ by $550 \mu \mathrm{m}$, a luminous flux of 48 lumen $(\mathrm{lm})$ and an average efficiency of $117 \mathrm{~lm}$ per watt.

To keep the design prototype-friendly, we decided to use a commercially available off-the-shelf plano-convex aspheric lens with circular symmetry for the first lens array. The lens has a focal length of $8 \mathrm{~mm}$ and a diameter of $10 \mathrm{~mm}$.

Simulation- and computer-aided design (CAD) files, available for the LED and for the first lens, were imported into our optical ray tracing simulation tool. Based on the two chosen components, the freeform curvature for the second lens was optimized through ray-tracing simulations, to obtain the highest collimation. After the curvature of the freeform lens was designed, the lens with circular symmetry was cut into a hexagon. 
The resulting design of the LED and the two lenses is shown in figure 1 . The total distance from the backside of LED to the front of the freeform lens is $20.8 \mathrm{~mm}$. The width of each hexagonal lens is $14.7 \mathrm{~mm}$, which is equal to the shortest period in the hexagonal LED array.
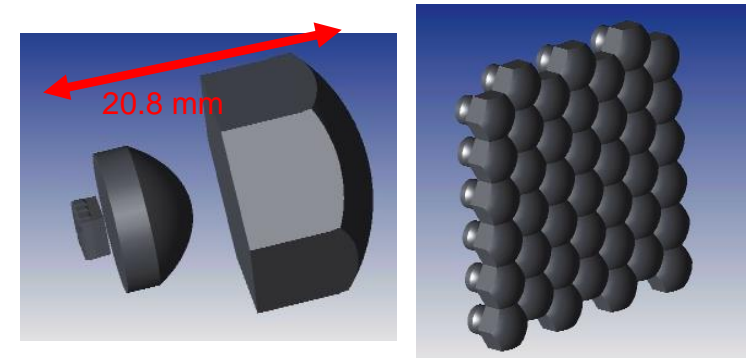

Figure 1. Design of the collimated backlight. Two planoconvex lenses are placed in front of each LED (left). By cutting the large freeform lenses into hexagons, they can be tiled into a lens array (right).

\section{Ray tracing simulation}

The configuration of one LED with two lenses is implemented in a ray tracing simulation program. The simulations results for one unit of the backlight are shown in figures 2 and 3 . The high degree of collimation in the horizontal and vertical directions is clearly visible in the conoscopic plot (figure 2). From the crosssection of the plot (figure 3), it can be observed that the degree of collimation is $2.8^{\circ}$ (FWHM).
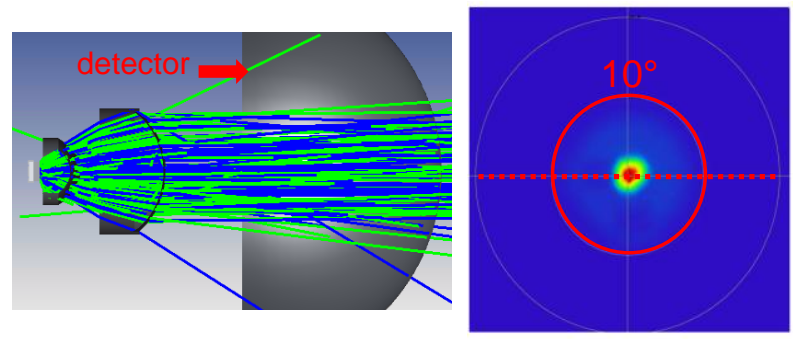

Figure 2: Ray tracing simulation for a single LED (white) with two lenses that collimate the light of the LED (left). The detector is indicated by a red arrow. The angular intensity profile is shown in a conoscopic plot (right).

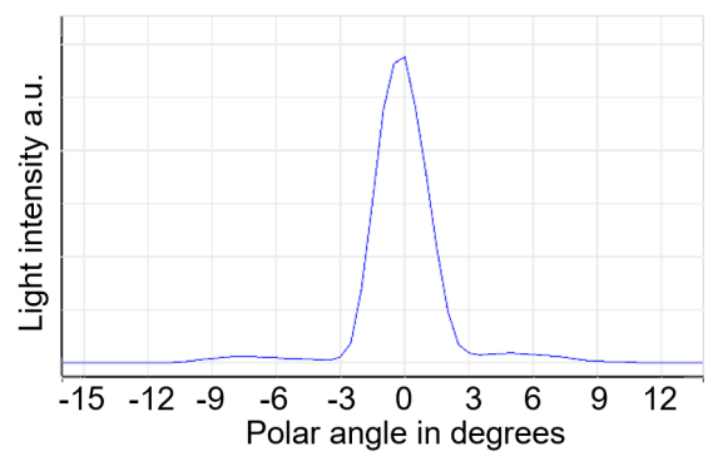

Figure 3: Cross section of the angular intensity profile in the horizontal plane of the LED array.
The simulated uniformity of a backlight consisting of 42 hexagonal unit cells is shown in figures 4 and 5. To obtain this result, the detector was placed at a distance of $10 \mathrm{~mm}$ from the hexagonal lens array. From the spatial intensity profile, slightly brighter regions can be noticed in the middle- and along the edges of each lens. The cross section reveals that uniformity is $72 \%$.

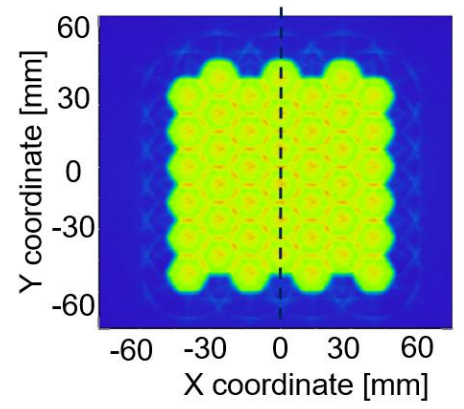

Figure 4: Numerical simulation of the area intensity profile of the designed backlight consisting of 42 LEDs and two lenses per LED.

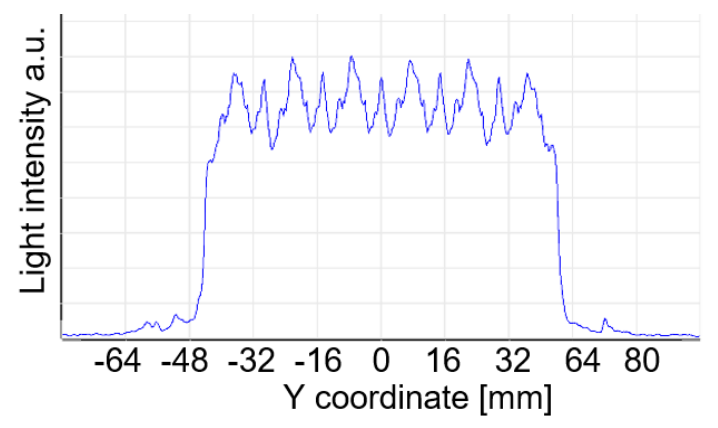

Figure 5: Cross section along the black dotted line in the spatial intensity profile shown in figure 4 .

\section{Prototype fabrication and characterization}

For the fabrication of the collimated backlight prototype, a set of LEDs was selected and mounted on a printed circuit board (PCB). Three frames were built, the first one to mount the PCB, the second one with 42 holes for mounting the off-the-shelf lenses, and the third frame to mount the hexagonal lens array. Each of the frames is designed to enable repositioning in 6 dimensions: $\mathrm{x}$ and $\mathrm{y}$ translation, height, rotation, tilt in $\mathrm{x}$ and $\mathrm{y}$ direction. The three frames are shown in figure 6.

The second lens array with the joint hexagonal lenses was made by the 3D printing company Luximprint which allows the costeffective prototype fabrication of custom optical components.

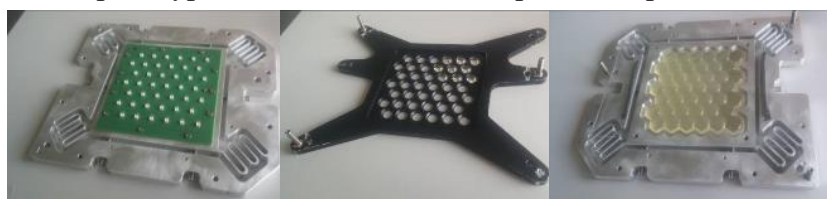

Figure 6: Three frames were built in order to position and fix the LEDs (left), first lenses (middle) and hexagonal lensarray (right) with respect to each other. 
From figure 6 it can be seen that the hexagonal lens array has a yellow tint. This is due to the UV-curing of the printed resin, which was relatively thick.

The collimation performance of the prototype was measured in two ways. The first measurement is based on the observation of the expansion of the spot as a function of the distance from the screen and resulted in a FWHM collimation angle of $6.9^{\circ}$. The second measurement is based on a goniometer (Eldim Ezcontrast) measuring the angular distribution for of a spot of 2 $\mathrm{mm}$ diameter. Multiple measurements were carried out in order to estimate the overall collimation of the backlight. The result of these measurements are shown in figure 7, the obtained FWHM collimation angle is about $6^{\circ}$.

The uniformity of the prototype is illustrated in figure 8. In order to achieve a sufficiently uniform illumination in which individual LEDs are no longer visible it is necessary to position the LC panel at a distance of $80 \mathrm{~mm}$, and use a weak diffusor. In this case the FWHM collimation angle is increased to $8.4^{\circ}$.

The measured intensity profile (left figure in figure 8 ) differs considerably from the simulated profile (figure 4) because there are black regions on the edge between adjacent hexagonal lenses. The origin of this deviation is explained in the next section.

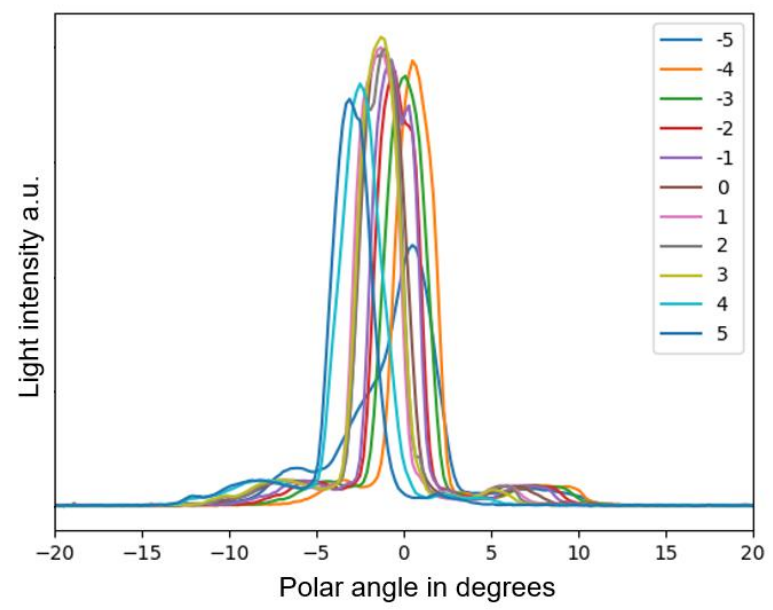

Figure 7: Angular intensity profiles measured with a goniometer, measurements at different $x$-coordinates in front of the same hexagonal lens ( $x$-coordinate from -5 $\mathrm{mm}$ to $+5 \mathrm{~mm})$.

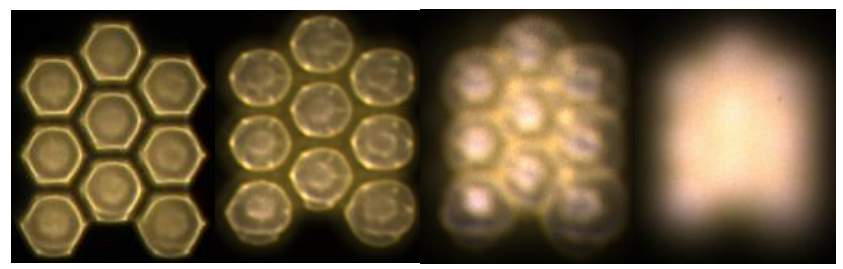

Figure 8: Measured spatial intensity variation of the prototype backlight when the screen is placed at $10 \mathrm{~mm}$ (left), $30 \mathrm{~mm}$ and $80 \mathrm{~mm}$ from the screen. With a weak diffusor, uniform illumination is obtained at $80 \mathrm{~mm}$ from the outgoing diffusor (right).

\section{Analysis of the fabricated lens array}

In order to explain the difference between the uniformity profile of the simulation and the prototype, the actual shape of the printed lens array was further investigated.

The surface of the printed lens array has been scanned in three dimensions using X-ray computed tomography (CT) scanning at the UGent Centre for X-ray computed tomography (UGCT). The result is a three dimensional CAD file, which can be used to compare the actual lens shape with the designed lens shape. Both lens shapes are shown in figure 9. The designed lenses have sharp edges, whereas the printed lenses have rounded edges, which is inherent to the used technology. The blue arrows in figure 9 show that the printed lenses have a higher curvature at a distance from the edges.

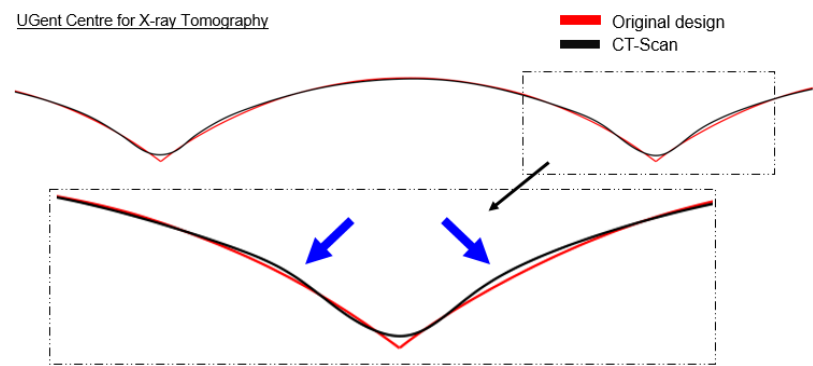

Figure 9: Comparison of the designed lens profile (red) with the actual printed prototype lens profile (black).

The CAD file obtained from the CT-scan was imported into our optical software program and a new simulation was performed based on this profile for the hexagonal lens array. The resulting spatial intensity profile is shown in figure 10 . This simulation indeed reproduces the dark regions observed in the measurements (figure 8).

Based on this simulation, the degree of collimation can be estimated. The angular intensity profile is shown in figure 11 and from the cross section the FWHM collimation angle is determined as $5.6^{\circ}$, which corresponds to the experimental values.

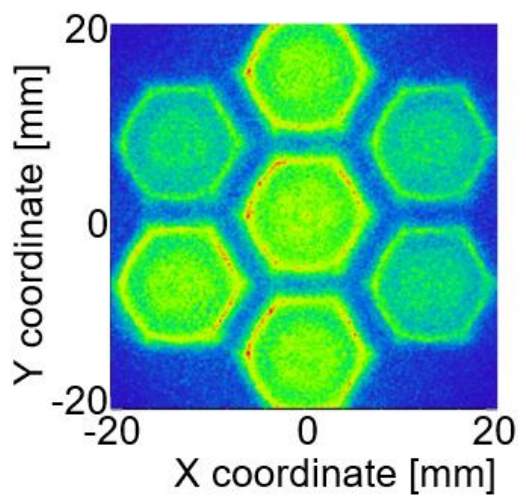

Figure 10: Simulation of the spatial intensity profile when the original hexagonal lens array is replaced by the CT profile of the printed lenses. The detector is again placed at $10 \mathrm{~mm}$ from the lens array. 


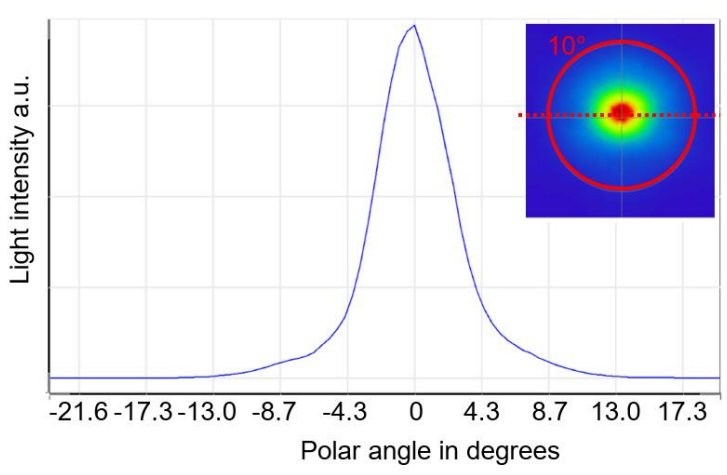

Figure 11: Simulation of the angular intensity profile based on the surface profile obtained from the CT-scan

\section{TNLCD Panel with collimated backlight}

Although the collimated backlight presented here has a limited uniformity, which originates from the rapid prototyping fabrication process, we have used it to verify if the display properties can be improved.

Two important issues of LCDs, especially LCDs based on the TN technology, are the viewing-angle dependency of colors and the light leakage (and reduced contrast ratio) at oblique viewing angles. Both issues are originating from the fact that light enters the liquid crystal panel from many different angles. An LC panel with collimated backlight does not suffer from these issues, as all the light is incident perpendicularly on the polarizers and on the liquid crystal panel, which is the optimum propagation direction for the TNLCD.

In order to create a larger viewing cone in combination with a collimated backlight, a diffusor can be placed in front of the LC panel (postdiffusor), facing the viewer. Figure 12 shows the measured color shift for different colors, for the collimated backlight unit (CBLU) with a postdiffusor (PD), compared to that of a standard backlight unit SBLU. It can be seen that an average color shift reduction of $79 \%$ was achieved when using a CBLU with PD compared to a standard backlight. The color shift is expressed as the distance in u'v' coordinates of the CIELUV color space.

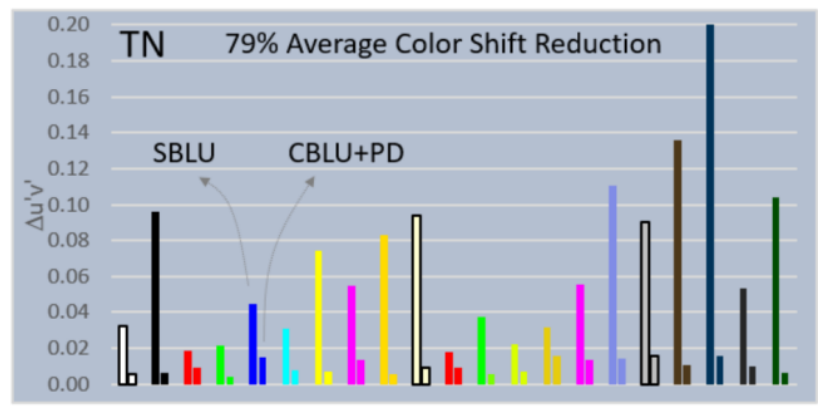

Figure 12: Maximum measured color differences for an off-the-shelf TN panel under different viewing angles $\left( \pm 60^{\circ}\right.$ horizontal, $\pm 35^{\circ}$ vertical) for a standard backlight unit (SBLU, left bar) and for the prototyped collimated backlight with a postdiffusor (CBLU+PD, right bar). The color of the bar represents the displayed color on the monitor.
For the normal observation direction, the contrast ratio for the CBLU with PD was a factor 4.2 times higher than for a standard backlight unit (SBLU). The collimated backlight with postdiffusor did not show any significant change in contrast ratio at $0^{\circ}$. However at a viewing angle of $50^{\circ}$, the contrast ratio was 10 times better.

\section{Conclusion}

A backlight with a high degree of collimation has been designed, based on a hexagonal array of small LEDs, an array of aspheric lenses and an array of freeform lenses. A prototype of the design was fabricated using an innovative and prototypefriendly manufacturing technology. Differences between ray tracing simulation results and optical measurements of the prototype could be explained by deviations between the fabricated lens array and the design. The performance of the collimated backlight was tested in combination with a TN panel and compared to a standard wide-emitting backlight. An average reduction of $79 \%$ in the color shift and a gain of a factor 10 in contrast ratio at a viewing angle of $50^{\circ}$ was observed.

\section{Impact of Your Research}

TNLCD panels suffer from color shift at large viewing angles but have excellent transmission. Using the approach of a collimated backlight in combination with a postdiffusor makes it possible to use a TN panel in situations where a high transmission and good oblique properties are required. The collimated backlight was realized based on an innovative 3D printing technology.

\section{Acknowledgements}

The authors want to thank the Centre for X-ray Tomography at Ghent University UGCT, for the use of the CT scanning facility. The authors also want to acknowledge the funding received from the Flemish Agency for Innovation and Entrepreneurship.

\section{References}

1. Tun-Chien Teng, Wen-Shing Sun, Li-Wei Tsen, WeiChung Chang. A slim apparatus of transferring discrete LEDs' light into an ultra-collimated planar light source. OE [Internet]. November 2013 [cited 2019 Nov 29]. 21(22):26972-26982. Available from:

https://www.osapublishing.org/oe/abstract.cfm?uri=oe-2122-26972 DOI: 10.1364/OE.21.026972

2. Yating Gao, Zhenyue Luo, Ruidong Zhu, Qi Hong, ShinTson Wu, Ming-Chun Li, et al. A high performance singledomain LCD with wide luminance distribution. JDT [Internet]. April 2015 [cited 2019 Nov 29]; 11(4):315-324. Available from:

https://ieeexplore.ieee.org/document/7060638/authors\#auth ors DOI: 10.1109/JDT.2015.2408993

3. Jui-Wen Pan, Chen-Wei Fan. High luminance hybrid light guide plate for backlight module application. OE [Internet]. October 2011 [cited 2019 Nov 29]. 19(21):20079-20087. Avaialble from: https://www.osapublishing.org/oe/abstract.cfm?uri=oe-1921-20079 DOI:10.1364/OE.19.020079

4. Edwin Diaz, Matthias Knobl. Prototyping illumination systems with stock optical components. [Internet] 2012 Oct [cited 2019 Nov 2019].24-27. Available from: https://www.edmundoptics.com/globalassets/knowledgecenter/articles/prototyping-illumination-systems-withstock-optical-components-en.pdf 